\title{
Protective Role of Selenium and High Dose Vitamin E against Cisplatin - Induced Nephrotoxicty in Rats
}

\author{
Asude Aksoy ${ }^{1 *}$, Aziz Karaoglu², Nusret Akpolat ${ }^{3}$, Mustafa Naziroglu ${ }^{4}$,Turkan \\ Ozturk $^{5}$, Zuhal Karaca Karagoz ${ }^{6}$
}

\begin{abstract}
Background: Cisplatin (CDDP) is one of the most active cytotoxic agents in the treatment of cancer. We investigated the effect of selenium (Se) with high dose vitamin $\mathrm{E}(\mathrm{VE})$ administration to prevent $\mathrm{CDDP}$-induced nephrotoxicity in rats. Materials and Methods: In this study, 40 female Wistar rats were randomly divided into five equal groups. The first group, which served as the control, was administered physiological saline (2.5 cc/day, 5 days) intraperitoneally (IP), while group A was administered cisplatin (6 mg/kg BW/ single dose) plus physiological saline IP. Groups B, C, D received IP five doses of Se $(1.5 \mathrm{mg} / \mathrm{kg} \mathrm{BW})$, and a high dose of $\mathrm{VE}(1000 \mathrm{mg} / \mathrm{kg} \mathrm{BW})(\mathrm{Se}-\mathrm{VE})$ in combination before, simultaneously, and after CDDP, respectively. The rats were sacrificed five days after CDDP administration. Plasma malondialdehide (MDA), glutathione peroxidase (GSH-Px), reduced glutathione (GSH), catalase, urea, creatinine levels, renal histopathological changes were measured. Results: The histopathological injury score, plasma levels of MDA, urea, creatinine were found to increase in group A compared to the control $(p<0.05)$, while plasma levels of GSH-Px, GSH and catalase decreased $(p<0.05)$. In contrast, plasma levels of MDA decreased $(p<0.05)$ in groups $B, C, D$, which were treated with SeVE, whereas levels of GSH-Px, GSH were found to increase only for group $D(p<0.05)$. Plasma urea, creatinine levels improved in the treatment groups compared to group $A(p<0.001)$. Histopathological changes caused by CDDP were also significantly improved after Se-VE treatment $(p<0.05)$. Conclusions: Oxidative stress increases with CDDP-induced nephrotoxicity in rats. Se-VE supplementation might thus play a role in the prevention of CDDP-induced nephrotoxicity in patients.
\end{abstract}

Keywords: Cisplatin-induced nephropathy - oxidative stress - vitamin E - selenium

Asian Pac J Cancer Prev, 16 (16), 6877-6882

\section{Introduction}

Cisplatin (CDPP) is currently at the cornerstone of the most important cytostatic agents in the treatment of solid tumors such as head and neck, ovarian, bladder, breast, lung and cervical cancers (Wang et al., 2012; Dimri et al., 2013; Erten et al., 2013; Gupta et al., 2013).

Although in the treatment of many cancers which use platinum-based chemotherapy, the clinical usefulness of this drug is limited by the development of nephrotoxicity, a side effect that may be produced in various animal models (Fuertes et al., 2002; Chirino et al., 2004). Therefore, high-doses of platinum administration have been restricted. Xenobiotics cause changes to kidney functions such as urine volume, creatinine clearance, changes to in glutathine status, and increased lipid peroxidation. Chemotherapeutic agents such as CDDP cross links DNA cause cytotoxic lesions such as the formation of free radicals from oxidative stress in neoplastic structure and regenerative cells. Reactive oxygen species (ROS) such as superoxide anion and the hydroxil radical create cellular injury and necrosis through several mechanisms including peroxidation of cell membrane lipids, protein denaturation, and even DNA damage can generate. The resulting ROS particles principally accumulate at the S3 segment of the proximal tubule in renal. Mitocondria continuously scavenges ROS through antioxidant enzymes such as superoxide dismutase (SOD), GSH, GSH-Px, and catalase formed by oxidative stress after platinumbased chemotherapy (Atessahin et al., 2005). While lipid peroxidation increases in the oxidative damage, a decrease is observed in the activity of anti- oxidant enzymes in the kidney tissues to prevent the integrity of biological membranes. With this logic, very different anti-oxidants such as Vitamin E (VE), Vitamin C, selenium (Se), carotenoids, ellagic acid, azadirachta indica, and ebselen along with cisplatin have been used to protect against nephrotoxicity in humans and experimental animals.

${ }^{1}$ Department of Medical Oncology, Medical Faculty, Firat University, ${ }^{2}$ Department of Medical Oncology, Medical Faculty, Dokuz Eylul University, ${ }^{3}$ Department of Pathology, Medical Faculty, Inonu University, ${ }^{4}$ Department of Biophysics, Medical Faculty, Suleyman Demirel University, ${ }^{5}$ Department of Medical Oncology, Medical Faculty, Karadeniz Teknik University, ${ }^{6}$ Department of Internal Medicine-Endocrinology, Elazig Regional Education and Research Hospital,Turkey*For correspondence: asudeaksoy@ hotmail.com 
Studies that have examined these agents report that an oxygen radical-scavenging system could reduce the extent of CDDP-induced nephropathy (Martinis et al., 2001; Arunkumar et al., 2012; Moneim et al., 2014).

Selenium, an essential dietary trace element, has been an important role in biological processes. Se, entering the GSH-Px structure, plays an important function in the activation of this enzyme. (Jones et al., 2014). Se has received particular attention as a result of many different studies on animals that have reported as highly efficient antioxidant. Low Se levels are shown to be related to thyroid, prostate, esophagus, colorectal, cervical, and bladder carcinomas in case-control studies. Se is also used to prevent chemotherapeutic agent-induced toxicity in preclinical and clinical studies (Arsenyan et al., 2014, Ji et al., 2014).

Vitamin $\mathrm{E}$ is an essential fat-soluble vitamin. VE is considered to think about the front line of defense against to lipid peroxidation, therefore its free radical-quenching activity preserves in the early stage of free radicals attacks in the cell membranes. (Kappus et al., 1992).

When used in high doses CDDP, levels of MDA were more increased, and levels of antioxidants were more decreased. Therefore, high levels of enzymatic and nonenzymatic antioxidants should be oxidized, and theirs of levels in renal tissue should be increased. (Fuertes et al., 2002, Weijl et al., 2004, Atessahin et al., 2005). The aim of this study is to evaluate the level of oxidative stress in CDDP-induced nephropathy and we investigated the effect of Se-VE administration to prevent CDDP-induced nephropathy in rats. The second aim of this study is to ascertain the most appropriate time of administration when using the Se-VE combination for the most beneficial effect as an antioxidant defense system.

\section{Materials and Methods}

\section{Chemicals}

The injecTable form of VE (dl- $\alpha$-tocopheryl acetate, Evigen ${ }^{\circledR}$ ) was obtained from Eras (Istanbul, Turkey). Cisplatin (10 mg/10 ml, Code $1876 \mathrm{~A})$ was purchased from Rhone Poulenc (Istanbul, Turkey). Other chemicals were supplied by Sigma Chemical Inc. (St. Louis, MO, USA), and all organic solvents and sodium selenite were supplied by Merck Chemical Inc. (Darmstadt, Germany). All reagents were stored at the same property. All reagents outside the phosphate buffers were prepared each day and stored in a refrigerator at $+4^{\circ} \mathrm{C}$. Phosphate buffers were stabilized at $+4{ }^{\circ} \mathrm{C}$ for about 1 month. The reagents were equilibrated at room temperature for $0.5 \mathrm{hrs}$. before the procedure.

\section{Animals and Study Groups}

The Medical Faculty Experimentation Ethics Committee of Firat University approved the experimental procedures of the study.

In this study, 40 adult female Wistar rats were obtained from Firat University Medical School, Experimental Research Centre, Elazig, Turkey. The animals were kept under standard laboratory conditions (12-hrs. light/12-hrs. dark and $24 \pm 3{ }^{\circ} \mathrm{C}$ with free access to water). All study procedures were in accordance with the Guide to the Care and Use of Laboratory Animals. During the study, the rats' bodyweights were assessed; the rats aged 12 weeks weighed 150-165 g. Each group consisting of eight rats was randomly divided into five groups.

Group K was administered physiological saline (2.5 cc/day, 5 days) IP.

Group A was administered a single dose of CDDP (6 $\mathrm{mg} / \mathrm{kg} \mathrm{BW}$ ) and four doses of physiologic saline (PS), IP. The animals were sacrificed 5 days after a single dose CDDP administration.

Group B were given five doses of Se $(1.5 \mathrm{mg} / \mathrm{kg} \mathrm{BW})$ and a high dose of VE (1000 mg/kg BW) (Se-VE) before CDDP administration and, they were administered a single dose of CDDP (6 mg/kg BW), IP, on the sixth day. The rats were sacrificed five days after CDDP administration.

Group C was administered a single dose of CDDP (6 $\mathrm{mg} / \mathrm{kg} \mathrm{BW}$ ) with administration concurrent started and continued for 5 days five doses of Se-VE. The rats were sacrificed five days after CDDP administration

Group D were administered a single dose of CDDP $(6 \mathrm{mg} / \mathrm{kg} \mathrm{BW})$ followed by five doses of Se-VE. The rats were sacrificed five days after CDDP administration.

At the end of the study on the tenth day, the animals were anaesthetized with ether for five minutes and decapitated. Blood samples were collected into containing $2 \%$ sodium oxalate anticoagulant coated tubes. The blood samples were centrifuged at $200 \mathrm{X}$ g for 5 minutes at $+4{ }^{\circ} \mathrm{C}$ to separate their plasma. The right kidney was removed and placed in $10 \%$ formaldehyde solution for histopathological analysis and stored at $-20{ }^{\circ} \mathrm{C}$ until analyzed. The left kidney was used for lipid peroxidation and for biochemical measurements.

\section{Laboratory Analysis}

In Plasma: MDA, GSH-Px, GSH, catalase, creatinine and urea levels were measured.

\section{Lipid Peroxidation Assay}

As indicators of lipid peroxidation, the levels of MDA were determined according to the reaction with thiobarbituric acid (TBARS), using the method Ohkawa's (Ohkawa et al., 1979) were expressed as $\mathrm{nmol} / \mathrm{ml}$.

\section{Reduced Glutathione (GSH), Glutathione Peroxidase (GSH-Px) and Catalase Assay}

The levels of GSH were measured at $412 \mathrm{~nm}$ in spectrophotometric, according to the method described in Sedlak and Lindsday (Sedlak et al., 1968) and was expressed as $\mu \mathrm{mol} / \mathrm{ml}$.

The levels of GSH-Px were measured at $412 \mathrm{~nm}$ in spectrophotometric, according to the method described in Lawrence and Burk (Lawrence et al., 1976) and was expressed as $\mathrm{U} / \mathrm{g}$ protein.

The levels of catalase were measured according to the method described in Aebi (Aebi , 1983) was expressed as $\mathrm{kU} /$ gram.protein.

\section{Determination of Serum Urea and Creatinine}

Serum levels of urea and creatinine were measured spectrophotometrically using commercial diagnostic kits 
Protective Role of Selenium and High Dose Vitamin E against Cisplatin-Induced Nephrotoxicty in Rats

ADVIA chemistry (Siemens Healthcare Diagnostics, Australia), according to the methods described in Tabacco (Tabacco et al., 1979).

\section{Histopathological Examinations}

After the sacrifice procedure, the tissues were fixed in $10 \%$ formalin, sectioned at $5 \mu \mathrm{m}$ and routinely stained with hematoxylin and eosin after being embedded in paraffin. The specimens were evaluated under light microscopy.

Histopathological evaluation criteria were identified: i) Tubular necrosis (TN). ii) Tubular dilatation (TD). ii) Interstitial nephritis (IN). iv) Basal membrane rupture (BMR)

Histopathological changes were evaluated in the framework of our scale (Ueda et al., 1986).

\section{Statistical Analysis}

Data was analyzed using SPSS statistical program (version 22.0 software, IBM, SPSS Inc. Chicago, Illinois, USA). All results are expressed as means \pm S.D. The Multiple Mann-Whitney U test was used to obtain standard deviation for mean values of all data. For differences between group means, data were analyzed using oneway ANOVA repeated measures. Significant values were assessed with Tukey's multiple range test. Determined $\mathrm{P}$-values of $<0.05$ were regarded as significant.

\section{Results}

Values detected in plasma MDA, GSH-Px, GSH, catalase, urea and creatinine are shown in Table 2.

\section{Changes in Plasma MDA Levels}

An indirect indicator of lipid peroxidation MDA levels was shown in Figure 1, Table 2.

Plasma MDA levels in group were high A compared to group $\mathrm{K}$, but no statistical significance existed ( $>0.05$ ).

Plasma MDA levels in groups $\mathrm{C}$ and $\mathrm{D}$ were low compared to group $\mathrm{K}$ and statistically significant $(\mathrm{p}<0.05)$.

Plasma MDA values of groups $\mathrm{B}, \mathrm{C}$, and $\mathrm{D}$ given $\mathrm{Se}-\mathrm{VE}$ (after treatment groups) compared to those given a single dose of CDDP in group A were significantly decreased (respectively, $\mathrm{p}<0.001, \mathrm{p}<0.01, \mathrm{p}<0.01$ ). Plasma MDA values of averages were group $\mathrm{A}>$ group $\mathrm{K}>$ group B $>$ group D> group C.

\section{Changes in Plasma Levels of Glutathione Peroxidase (GSH-Px)}

Levels of GSH-Px are shown in Figure 2 and Table 2. Plasma GSH-Px values of averages were group D> group $\mathrm{C}>$ group $\mathrm{B}>$ group $\mathrm{K}>$ group $\mathrm{A}$.

Plasma GSH-Px values in group A were low compared to $\mathrm{K}$ group, but no statistical significance existed ( $\mathrm{p}>0.05)$. Plasma GSH-Px values after administration of a single dose of CDDP and five doses of Se-VE for five days in group D compared to a single dose of CDDP in group A were found to be statistically significantly high $(\mathrm{p}<0.05)$.

Changes in Plasma Levels Reduced Glutathione (GSH)

Levels of GSH are shown in Figure 3, Table 2. Plasma GSH value of averages were group $\mathrm{D}>$ group $\mathrm{C}=\mathrm{B}>$ group $\mathrm{K}>$ group $\mathrm{A}$.

Plasma GSH values after administration of a single dose of CDDP and five doses of Se-VE for five days in group D compared to a single dose of CDDP in group A

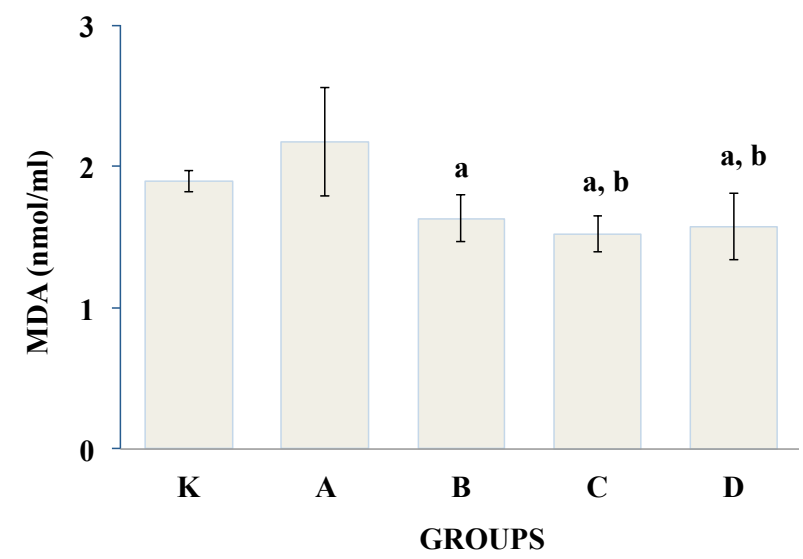

Figure 1. Distribution Plasma MDA between the Groups. (a): $\mathrm{p}<0.001$, Groups compared to group A, (b): $\mathrm{p}<0.01$, Groups compared to group $\mathrm{K}$

Table 1. Plasma Levels of Oxidative Factors

\begin{tabular}{lccccc}
\hline Studies Groups $(\mathrm{n}=8)$ & $\mathrm{K}$ & $\mathrm{A}$ & $\mathrm{B}$ & $\mathrm{C}$ & $\mathrm{D}$ \\
\hline MDA $(\mathrm{nmol} / \mathrm{ml})$ & $1.89 \pm 0.07$ & $2.17 \pm 0.34$ & $1.63 \pm 0.16$ & $1.52 \pm 0.13$ & $1.57 \pm 0.24$ \\
GSH-Px $(\mathrm{IU} / \mathrm{g}$ prot.) & $7.23 \pm 2.08$ & $5.59 \pm 1.25$ & $7.45 \pm 1.32$ & $7.68 \pm 2.62$ & $8.33 \pm 1.83$ \\
GSH $(\mu \mathrm{mol} / \mathrm{ml})$ & $0.62 \pm 0.04$ & $0.60 \pm 0.06$ & $0.64 \pm 0.10$ & $0.64 \pm 0.06$ & $0.71 \pm 0.08$ \\
Catalase $(\mathrm{kU} / \mathrm{mg}$ prot$)$ & $6.32 \pm 1.07$ & $5.36 \pm 0.78$ & $6.85 \pm 1.88$ & $7.88 \pm 1.91$ & $6.62 \pm 1.06$ \\
Urea $(\mathrm{mg} / \mathrm{dl})$ & $31.5 \pm 4.4$ & $181.37 \pm 106.68$ & $70.25 \pm 69.09$ & $67.62 \pm 43.51$ & $72.25 \pm 76.49$ \\
Creatinine $(\mathrm{mg} / \mathrm{dl})$ & $0.41 \pm 0.2$ & $1.77 \pm 0.95$ & $0.97 \pm 0.60$ & $7.68 \pm 2.62$ & $0.65 \pm 0.46$ \\
\hline
\end{tabular}

MDA: Malondialdehit, GSH-Px: Glutathione Peroxidase, GSH: Reduced Glutathione

Table 2. Histopathologic Changes in Groups

\begin{tabular}{lllll}
\hline Studies Groups & \multicolumn{1}{c}{ TN } & \multicolumn{1}{c}{ TDL } & \multicolumn{1}{c}{ IN } & \multicolumn{1}{c}{ BMR } \\
\hline K & $0.12 \pm 0.35$ & 0 & 0 & 0 \\
$\mathrm{~A}$ & $2.5 \pm 0.75 \mathrm{~b}$ & $1.37 \pm 0.51 \mathrm{~b}$ & $0.37 \pm 1.06 \mathrm{a}$ & $1.37 \pm 0.91 \mathrm{~b}$ \\
$\mathrm{~B}$ & $1.42 \pm 0.78$ & $1.28 \pm 0.75 \mathrm{a}$ & $0 \mathrm{c}$ & $0.85 \pm 0.69$ \\
$\mathrm{C}$ & $1.62 \pm 0.91 \mathrm{~b}$ & $1.25 \pm 0.88 \mathrm{a}$ & $0.12 \pm 0.35$ & $0.87 \pm 0.83$ \\
$\mathrm{D}$ & $0.14 \pm 0.37 \mathrm{~d}$ & $0.85 \pm 0.37 \mathrm{~b}$ & $0 \mathrm{c}$ & $0 \mathrm{c}$ \\
\hline
\end{tabular}

BMR: Basal Membrane Rupture , TDL: Tubular Dilatation, TN Tubular Necrosis:, IN: Interstitial Nephritis. (a) p < 0.05, (b) p<0.01, Groups compared to group $\mathrm{K}$. (c) $\mathrm{p}<0.05$, (d) $\mathrm{p}<0.001$., Groups compared to group A 


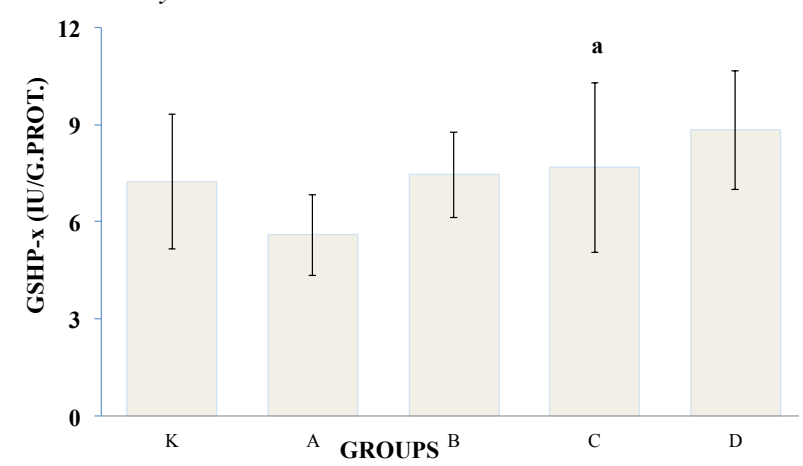

Figure 2. Distribution of Plasma GSH-Px between the Groups. (a): $p<0.001$, Groups compared to group A

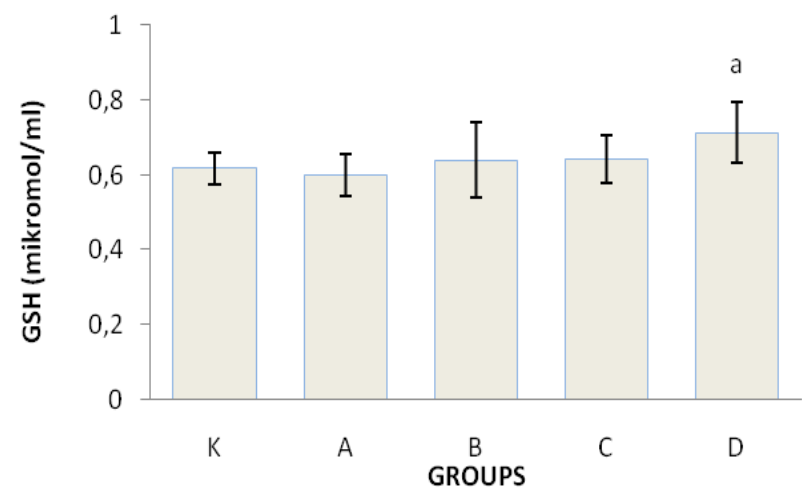

Figure 3. Distribution of Plasma Reduced GSH between the Groups. (a): $p<0.001$, Groups compared to group A

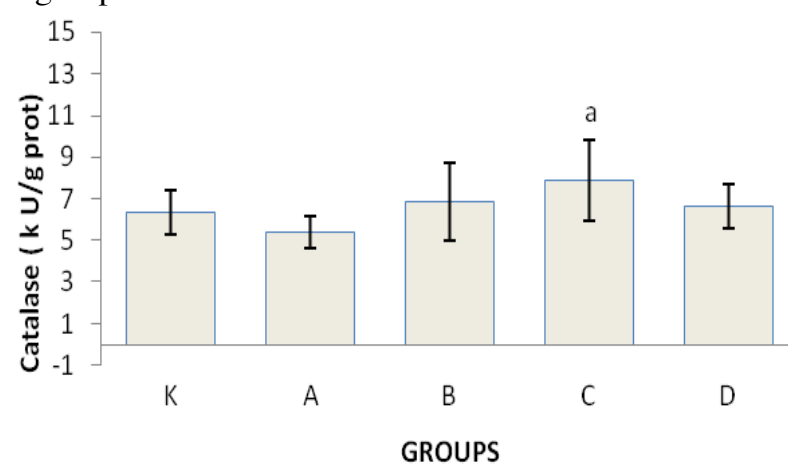

Figure 4. Distribution of Plasma Catalase between the Groups. (a): $p<0.001$, Groups compared to group A

were found to be statistically significantly high $(\mathrm{p}<0.05)$.

\section{Changes in Plasma Levels Catalase}

Levels of catalase are shown in Figure 4 and Table 2. Plasma catalase values of averages were group $\mathrm{C}>$ group $\mathrm{D}>$ group $\mathrm{B}>$ group $\mathrm{K}>$ group $\mathrm{A}$. When plasma catalase values in group $\mathrm{A}$ were compared to $\mathrm{K}$ group, they were high, but there was no statistical significance $(p>0.05)$.

Plasma catalase values of CDDP administration concurrent with five doses of Se-VE for five days in group C compared to group A were found to be statistically significantly high $(\mathrm{p}<0.01)$.

\section{Changes in Plasma Levels of Urea and Creatinine}

Means of the clinical biochemical indicators of CDDPinduced nephrotoxicity urea and creatinine levels are shown in Table 2.

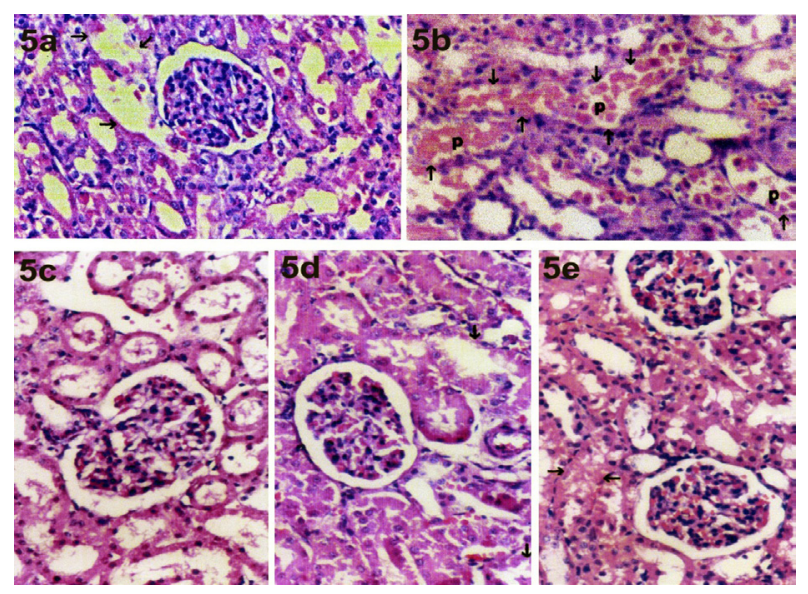

Figure 5. Histopathological Appearance of the Groups (Heomotoksilen-EosinX200). a)Histopathological appearance of the control group normal glomeruli and tubules of the kidney structures. b). Histological appearance of the group treated with CDDP. In the proximal tubule necrosis due to severe damage (arrows) and epithelial desquamasyo (d) are shown. c) Histological appearance of the B group given Se-VE before CDDP administration. Focal necrosis in proximal tubule epithelial cells, regenerative changes seen in the tubule epithelial cells.d) Histopathological appearance of the C group CDDP with administration concurrently starded Se-VE. Seen focal necrosis ( arrows) in proximal tubule epithelial cells. e) Histological appearance of the D group given and continuing after CDDP $\mathrm{Se}-\mathrm{VE}$ administration. In the proximal tubule mild vacuolar degeneratives (arrows) and regenaratives changes are shown.

When plasma urea and creatinine values in group A were compared to group $\mathrm{K}$, a high statistical significance was evident $(\mathrm{p}<0.001)$.

Plasma urea and creatinine values of groups B, C, and $D$ were higher than group $K$ but there were no statistically significant differences $(\mathrm{p}>0.05)$.

Comparisons between values of plasma MDA, GSHPx, reduced GSH, catalase, urea, and creatinine following administration of Se-VE at different times in groups B, $\mathrm{C}$, and $\mathrm{D}$ showed no statistically significant differences $(\mathrm{p}>0.05)$.

\section{Histopathological Results}

Histopathological damage to TN, TDL, IN, BMR parameters were scored as shown in Table 1.

The histopathological examination revealed minimal TNA findings in the control group. Of all the groups, the most apparent histopathological damage was observed in the group administered CDDP and PS. Histopathological damage findings in the treatment group showed varying degrees of reduction. B, C, D respectively describes the appearances of histopathologic damage, as shown Figure 5.

\section{Discussion}

As a result of developments in cancer treatment with prolongation and increased probability of recovery from cancer, in the last decade, attentions have focused on the protection of life from chemotherapeutic agents induced toxicity (Ferlay et al., 2014).

Although CDDP is frequently used in treatment 
of solid organ cancer, side of effects its such as dosedependent and cumulative nephrotoxicity limits clinically use of this agent.

Many opinions have been reported to explain the pathophysiology of CDDP-induced nephrotoxicity.The most widely accepted formation of free radicals during the use of CDDP. ROS radicals are the result of the development of acute renal injury (Atessahin et al., 2005). The occurrence of renal injury is heavily dependent on renal tubule damage. Glutamine is synthesized in the renal proximal tubule from which ammonia is secreted combined with $\mathrm{H}+$ and ammonia ions. Hydrolyzed products of CDDP interact much faster with molecules containing sulfur. Sulfhydryl groups interacting with CDDP causes a reduction in some mechanisms of cellular protection, resulting in low levels of GSH (Ghorbani et al., 2012). However, some publications suggest these substances don't play a protective role (Antunes et al., 2001).

GSH-Px is mainly responsible in normal conditions for detoxification of $\mathrm{H} 2 \mathrm{P} 2$ in cells. Initiation and progression inhibiting lipid peroxidation and tetrameric structure containing $4 \mathrm{Se}$ atoms within GSH-Px is a cytosolic enzyme. GSH-Px prevents to damage in the phagocytic cells resulting free radical peroxidation together with other antioxidants during aerobic respiration. GSH-Px in erythrocyte is also the most effective antioxidant against oxidative stress. GSH-Px activity in erythrocyte is an index of Se status. The between amount of plasma Se and GSH-Px activity has a direct relationship. GSHPx activities have been shown to increase from $30 \%$ to $1400 \%$ diet by administering Se in humans. In lung, lens, erythrocytes and platelets GSH-Px activity are a higher proportion than in other tissues (Jerome-Morais et al., 2012). Increased levels of GSH-Px are protective against oxidative damage in with precancer or cancer patients during their lifetime (Zhuo et al., 2009; Ha et al., 2014). VE in its structure the hydrogen ions give to hydroxyl radicals. In this way, VE acts as a protection against lipid peroxidation of cellular membranes.

As the most important system chain-breaking antioxidant in body tissues, VE blocks oxidation of unsaturated fatty acids in the cell membrane. However, the use of high doses of VE was not observed in acute or chronic toxicity. Studies have confirmed that 600-3200 IU / day doses VE can be taken for 3 weeks to 6 months without mutagenic, teratogenic, and carcinogenic side effects (Kappus et al., 1992).

GSH, GSH-Px, and other antioxidant enzyme levels and activities decreased due to consumption in CDDPinduced nephropathy. The activity of those enzymes would be expected to increase by giving the outside the reinforcement of these element. Both VE and Se suppress oxidative stress (Brigelius-Flohe et al., 2009). Thus, the power of the antioxidant system will achieve the desired outcome.

If necessary, ROS radicals are scavenged via antioxidant enzymes such as GSH-Px, catalase, reduced GSH in mitochondria viable cell structures. As the toxic effects of CDDP result in renal proximal tubule mitochondria, it is difficult to produce enough antioxidant enzymes to capture ROS radicals. Consequently, the levels of those antioxidations are detected a reduction in the serum. Moreover, it is determined that reduced levels of GSH-Px, GSH, catalase in plasma after CDDP administration, and those parameters also determined significantly increased after Se-VE in our study. Se-VE was able to renovate biosynthesis of GSH-Px, GSH, and catalase such as vitamin $\mathrm{A}$, vitamin $\mathrm{C}$, curcumine, resveretrol and VE depending on the antioxidant effects (Kappus et al., 1992; Yokozawa et al., 2000; Martinis et al., 2001; Gurocak et al., 2013; Ji et al., 2014; Zhao et al., 2014).

Our study also similarly determined that alterations in serum creatinine and urea levels in CDDP-induced nephropathies as in the studies by Atessahin (Atessahin et al., 2005), and Moneim (Moneim et al., 2014). However, the administration of Se-VE before, simultaneously, or after CDDP significantly improved renal function. The histopathological changes were seen in the more tubular necrosis and dilation structure, interstitial nephritis, rupture of the basement membrane due to CDDP's and accumulation of formed thanks to its formed ROS radicals in the proximal tubule epithelial cells in CDDP-induced nephrotoxicity. An increase of producing of ROS such as superoxide anion and hydroxyl radicals initiates renal lipid peroxidation. The severity of the degree of oxidative stress has a direct relationship with the amount of ROS radicals. Free radicals initiate lipid peroxidation reactions known as acting on polyunsaturated fatty acids and consisting of phospholipid membrane cells. In our study, oxidative stress was found to play an important role in the pathophysiology of CDDP-induced nephrotoxicity. CDDP administration was found to increase lipid peroxidation. The levels of lipid peroxidation product MDA, which were found to increase after CDDP administration. The levels of GSH-Px, GSH and catalase, which play an important role in the antioxidant system, were found to decrease after CDDP administration. The groups treated by supplementation of Se-VE were been found to have decreased CDDP-induced lipid peroxidation. The groups treated by supplementation of Se-VE showed decreased CDDP-induced nephrotoxicity. However, supplementation of Se-VE after the CDDP administration was found to be more useful in reducing CDDP-induced nephrotoxicity; a significant difference was observed when Se-VE was administered at different times to CDDP treatment. Therefore, supplementation of Se-VE after CDDP could be said to be more advantageous.

In conclusion: $i$ ) Oxidative stress plays an important role in the pathophysiology CDDP-induced nephrotoxicity. ii) Lipid peroxidation increases after CDDP. The levels of MDA products of lipid peroxidation increase after CDDP. The levels of GSH-Px, GSH, catalase play an important role in the antioxidant system, decrease after CDDP. iii) Use of Se-VE decreases CDDP-induced lipid peroxidation and CDDP-induced nephrotoxicity. Use of $\mathrm{SeVE}$ after CDDP is likely to be more useful for reduction nephrotoxicity in patients treated with CDDP in a clinical setting. 


\section{Acknowledgements}

The authors declare that they have no conflict of interest relating to the publication of this manuscript

\section{References}

Abdel Moneim AE, Othman MS (2014). Azadirachta indica attenuates cisplatin-induced nephrotoxicity and oxidative stress. Biomed Res Int, 647131.

Aebi H (1983). Methods in enzymatic analysis. in catalase, bergmeyer HU (Ed.), academic press, New York, 3, 276-86.

Antunes LM, Darin JD, Bianchi N de L. (2001). Effects of the antioxidants curcumin or selenium on cisplatin-induced nephrotoxicity and lipid peroxidation in rats. Pharmacol Res Feb, 43, 145-150.

Arsenyan P, Paegle E, Domracheva I, Gulbe A, Kanepe-Lapsa I (2014). Shestakova i. selenium analogues of raloxifene as promising antiproliferative agents in treatment of breast cancer. Eur J Med Chem, 87, 471- 83.

Arunkumar PA, Viswanatha GL, Radheshyam N, et al (2012). Science behind cisplatin-induced nephrotoxicity in humans: a clinical study. Asian Pac J Trop Biomed, 2, 640- 44.

Atessahin A, Yilmaz S, Karahan I, et al (2005). Effects of lycopene against cisplatin-induced nephrotoxicity and oxidative stress in rats. Toxicol, 212, 116-23.

Brigelius-Flohe R, Kipp A (2009). Glutathione peroxidases in different stages of carcinogenesis. Biochim Biophys Acta, 1790, 1555- 68 .

Chirino, YI, Hernandez-Pando R, Pedraza-Chaverri J (2004). Peroxynitrite decomposition catalyst ameliorates renal damage and protein nitration in cisplatin-induced nephrotoxicity in rats. BMC Pharmacol, 4, 20-29.

Dimri K, Pandey AK, Trehan R, et al (2013). Conventional radiotherapy with concurrent weekly Cisplatin in locally advanced head and neck cancers of squamous cell origin-a single institution experience. Asian Pac J Cancer Prev, 14, 6883-88.

Erten C, Demir L, Somali I, et al (2013). Cisplatin plus gemcitabine for treatment of breast cancer patients with brain metastases; a preferential option for triple negative patients? Asian Pac J Cancer Prev, 14, 3711-17.

Ferlay J, Soerjomataram I, Dikshit R, et al (2014). Cancer incidence and mortality worldwide: Sources, methods and major patterns in GLOBOCAN 2012, Int J Cancer, 136, 359-86.

Fuertes MA, Castilla J, Alanso C, et al (2002). Novel concepts in the development of platinum antitumor drugs. Current Medicinal Chemistry- Anticancer Agents, 2, 539-51.

Jerome-Morais A, Wright ME, Liu R, Yang W, et al (2012). Inverse association between glutathione peroxidase activity and both selenium-binding protein 1 levels and Gleason score in human prostate tissue. Prostate, 72, 1006-12

Ji J, Liu J, Liu H, Wang Y (2014). Effects of fermented mushroom of cordyceps sinensis, rich in selenium, on uterine cervix cancer. Evid Based Complement Alternat Med, 2014, 173180 .

Jones DP, Radi R (2014). Redox pioneer: professor helmut sies. Antioxid Redox Signal, 21, 2459-68

Ghorbani A, Omidvar B, Parsi A (2013). Protective effect of selenium on cisplatin induced nephrotoxicity: A double blind controlled randomized clinical trial. J Nephropathol, 2, 129-34.

Gupta RK, Singh N (2013). Morinda citrifolia (Noni) alters oxidative stress marker and antioxidant activity in cervical cancer cell lines. Asian Pac J Cancer Prev, 14, 4603-6.

Gurocak S, Karabulut E, Karadag N, et al (2013). Preventive effects of resveratrol against azoxymethane induced damage in rat liver. Asian Pac J Cancer Prev, 14, 2367-70.

Ha YS, Lee GT, Kim Y H, et al (2014). Decreased seleniumbinding protein $1 \mathrm{mRNA}$ expression is associated with poor prognosis in renal cell carcinoma. World J Surg Onco, 12, 288.

Wang J, Liu F, Huang DX, et al (2012). Post-operative treatment with cisplatin and vinorelbine in Chinese patients with nonsmall cell lung cancer: a clinical prospective analysis of 451 patients. Asian Pac J Cancer Prev, 13, 4505-10.

Kappus H, Diplock AT (1992).Tolerance and safety of vitamine E: a toxicological position report. Free Rad Biol Med, 13, 55-74.

Lawrence RA, Burk RF (1976). Glutathione peroxidase activity in selenium-deficient rat liver. Biochem Biophys Res Commun, 71, 952-58.

Martinis BS, Bianchi M de P (2001). Effect of vitamin c supplementation against cisplatin-induced toxicity and oxidative DNA damage in rats. Pharmacol Res, 44, 317-20.

Ohkawa H, Ohishi N, Yagi K (1979). Assay for lipid peroxides in animal tissues by thiobarbituric acid reaction. Anal Biochem, 95, 351-58.

Sedlak J, Lindsay RHC (1968). Estimation of total, protein bound and non-protein sulfhydryl groups in tissue with Ellmann's reagent. Anal Biochem, 25, 192-05.

Tabacco A, Meiattini F, Moda E, et al (1979). Simplified enzymic/colorimetric serum urea nitrogen determination. Clinical Chemistry, 25, 336-37.

Weijl NI, Elsendoorn, TJ, Lentjes, EG, et al (2004). Supplementation with antioxidant micronutrients and chemotherapy-induced toxicity in cancer patients treated with cisplatin-based hemotherapy: a randomised, double blind, placebo-controlled study. Eur J Cancer , 40, 1713-23.

Ueda T, Mihara Y, Yoshimina K (1986). Mechanism of cisplatinium neprotoxicity. Jpn J Nephrol, 28, 940-42.

Yokozawa T, Liu ZW (2000).The role of ginsenoside-Rd in cisplatin-induced acute renal failure. Ren Fail, 22, 115-27.

Zhao JA, Peng L, Geng CZ, et al (2014). Preventive effect of hydrazine curcumin on carcinogenesis of diethyl nitrosamine-induced hepatocarcinoma in male SD rats. Asian Pac J Cancer Prev, 15, 2115-21.

Zhuo P, Diamond AM (2009). Molecular mechanisms by which selenoproteins affect cancer risk and progression. Biochim Biophys Acta, 1790, 1546-54. 